\title{
A Reproducible and Scalable Method for Producing Fluorescent Polystyrene Nanoparticles
}

Dávid Bartoš ${ }^{1}$, Lu Wang ${ }^{* 1}$, Andy S. Anker ${ }^{1}$, Morten Rewers ${ }^{2}$, Olivia Aalling-Frederiksen ${ }^{1}$, Kirsten M. $\varnothing$. Jensen ${ }^{1}$, and Thomas Just Sørensen ${ }^{* 1}$

Address:

1. Nano-Science Center \& Department of Chemistry, University of Copenhagen, Universitetsparken 5, 2100 Copenhagen $\varnothing$, Denmark

2. FRS-Systems ApS

Hovedgaden 20, 4621 Gadstrup, Denmark

Email:

Lu Wang* - luwa@chem.ku.dk;

Thomas Just Sørensen* - tis@chem.ku.dk

* Corresponding author

Abstract

To fully exploit nanomaterials as components for advanced materials, they have to be made in a reproducible manner. As components, the nanomaterials do not have to be perfectly homogeneous nor uniform, but must adhere to the specifications required for the target application e.g. as building block for fluorescent composite materials. Here, polystyrene nanoparticles (PS NPs) were fabricated from an emulsion of PS/toluene in water using various surfactants, and purified via dialysis in a simple procedure. The synthesis process was carried out at room temperature, without hazardous chemicals, and with a workload of 5 hours. All relevant parameters - surfactant type, component concentrations, solvent volumes, polymer chain length, sonication time - were varied and the effect on the size of the resulting PS NPs were determined. These investigations were performed to evaluate the limits for production of PS NPs with comparable properties. A robust PS NP synthesis procedure was developed, repeated, and tested by three independent researches. The procedure was up-scaled to prove the applicability of the method and the NPs were prepared with four different hydrophobic dyes. All products were found to be comparable, and it was concluded that the method reported here can provide PS NPs with or without dye dopants, and that it provides access to PS NPs with an average diameter of $25 \mathrm{~nm}$ in a reproducible size distribution.

Keywords: fluorescent materials; fluorescent nanoparticles; nanomaterials; nanoparticle synthesis; polystyrene nanoparticles;

\section{Introduction}

With an interest in functional fluorescent materials,[1-5] we have followed the development of fluorescent nanomaterials that has seen a great development since the coining of the term in the 1950s.[6] Today, luminescent quantum dots, fluorescent polymer nanoparticles (NPs), luminescent nanodiamonds, and lipid-based nano-assemblies are used in various applications from display technologies for household electronic products and smartphones to cancer theranostics.[7-16] Most prevalent in industrial use are quantum dots, or specifically fluorescent semiconductor nanoparticles, 
which over three decades have been engineered to the point where bulk production (hundreds of kilograms to tons) of emitters with colours all across the spectrum are possible.[7, 17-19] This has led to an industrialization of the material and products like quantum-dot TV,[20] and quantum-dot based solar cells.[21] A similar evolution of other fluorescent nanomaterials is yet to happen, and there are only few industrial fluorescent nanomaterials.

Fluorescent nanomaterials offer high brightness and photostability in components that on the macroscale distribute as if they were molecules. Thus, they give rise to homogeneous fluorescent signals from bulk materials. The brightest nanomaterials are based on dye doped nanoparticles, where Reisch and Klymchenko have created super bright fluorescent nanomaterials by doping polymer nanoparticles with rhodamines ion paired with bulky fluorinated anions.[22-24] While this method is limited by aggregation-indused quenching,[25, 26] several high performing materials have been reported.[27-29] Very recently, fluorescent materials with a record brightness have been reported by Flood and Laursen. The technology, based on small-molecule ionic isolation lattices (SMILES) surpass all other organic dye-based fluorescent materials, $[25,30]$ and has recently been used to make fluorescent nanomaterials.[31] All these ultrabright nanomaterials constitute great advances in nanoscience, yet for nanotechnological applications, controlled manufacture is still needed.

Quantum dots are often made from heavy metals which, despite the excellent photophysical properties, limits applications. [32-35] Fluorescent materials for biological applications has to pass USP class IV certification (U.S. Pharmacopeia), which limits the amount of hazardous material that can be included. Here, polymer based nanoparticles synthesized from certified starting materials with low intrinsic toxicity and good biocompatibility have a significant advantage. Polystyrene (PS) in particular is a good material which is widely used and the synthesis of PS NPs has received a great deal of attention as a model system for developing polymer nanotechnology.[36, 37] By functionalizing the NPs with dyes or biological molecules, PS NPs have been used in optical sensors for metabolism tracking, [38] and commercial PS beads doped with fluorophores are sold at high prices.

PS NPs are normally synthesized by the microemulsion polymerization method, by polymerizing the monomer styrene directly in the emulsion. [39-47] In these systems an emulsifier, an initiator, and a cross-linker are also needed.[48] Briefly, the NP fabrication occurs in the emulsion at temperatures in the range of $65-80{ }^{\circ} \mathrm{C}$, which is kept for $6-8$ hours in an $\mathrm{N}_{2}$ atmosphere.[44, 45] The emulsion polymerization has been used to synthesize polymer particles for more than 50 years, and it has been shown in the scientific literature that this method allows for production of batches of monodisperse PS NPs smaller than $100 \mathrm{~nm}$. However, these beads may contain residual contaminant from the synthesis, and to our knowledge an industrial supply of dye doped PS NP is still to emerge. To remedy this, we here explored an alternative PS NP production process. By starting from PS, we have no additives, which should ensure USP class IV certification.

In this work, we report a standard method for making dyes doped PS NPs. We fabricated PS NPs using a purely physical method directly from bulk PS polymer. We used a surfactant enabled microemulsion method based on toluene/water mixtures.[49-51] There are several parameters that change the outcome of the synthesis:

- Surfactant type

- Surfactant concentration

- Ratio of toluene to water

- PS in toluene concentration

- PS molecular weight

- Sonication effect (power) in each refinement 
- Number of refinements (sonication cycles)

- Waiting time between refinements

- Addition of hydrophobic additives (dyes)

We chose to vary all above to thoroughly investigate how each factor influence the PS NPs produced using our protocol. We mapped the influence of each parameter, and tested the repeatability of the fabrication protocol. Finally, we explored the potential for industrial scale production by increasing the scale five times. As part of the study, we included four different hydrophobic dyes and found that they were all included in the PS NPs, and all maintained their photophysical properties.

\section{Results}

\section{Protocol for polystyrene nanoparticle preparation}

Polystyrene nanoparticles (PS NPs) were prepared with the sonication microemulsion method.[52-56] PS beads ( $\mathrm{Mw}, 35,000,280,000$ or 350,000 , with or without dye) were dissolved in toluene at concentrations between 0.5 and $2 \mathrm{w} / \mathrm{w} \%$, and a surfactant (sodium dodecyl sulphate (SDS), cetrimonium bromide (CTAB), Triton X-100 or Synperonic F108) in a concentration between $1 \mu \mathrm{g} / \mathrm{ml}$ and $20 \mathrm{mg} / \mathrm{ml}$ was dissolved in water. The NP synthesis was performed using a standardized method as shown in Scheme 1. The two phases were mixed by sonication for $1 \mathrm{~min}$ (cycling the sonication with 1 second on and 1 second off) using an immersion sonicator probe $(7 \mathrm{~mm} \varnothing)$ and sonication power of $70 \mathrm{~W}$. Each 1 minute sonication cycles is also known as a refinement.

Each refinement was followed by stirring in a closed vessel at with a 20 by $5 \mathrm{~mm}$ bar magnet at 300 rpm for 1 hour. This emulsification was done in a series of 5 refinements. Subsequently, the toluene was evaporated by stirring ( $300 \mathrm{rpm}$ ) at room temperature for 2 days. Finally, the PS NPs was isolated and purified by dialysis for 2 days, with 5 changes of the water. Several parameters affect this process and we set out to explore which were critical for obtaining similar products.

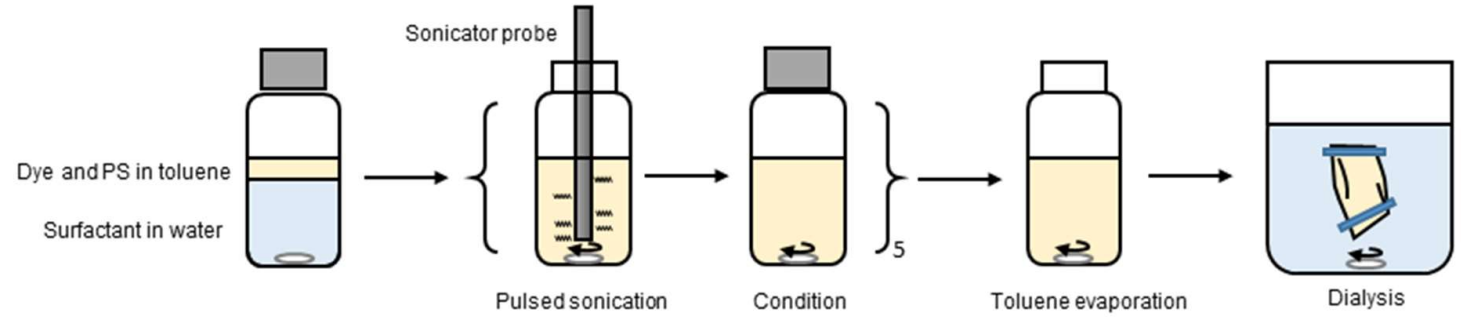

Scheme 1. Standard synthesis procedure for the PS NPS.

\section{Probing the effects of each parameter}

\section{Effect of the surfactant concentration}

Surfactants are used to stabilize the toluene/PS emulsion in the NP synthesis. As the concentration of the surfactant is expected to change the area of the water/toluene interface, NPs was expected to show different size distributions at different surfactant levels.[57, 58]

We prepared PS NPs using different amounts of the surfactant sodium dodecyl sulfate (SDS) (from 1 $\mu \mathrm{g} / \mathrm{ml}$ to $20 \mathrm{mg} / \mathrm{ml}$, see the synthesis procedure in experimental section). Figure 1 shows SEM images of six different batches of PS NPs with varying concentrations. Diameters of more than 200 NPs in each image were measured using the ImageJ software. The size distribution statistics were plotted and included in Figure 1. The size distribution of the particles does change with the concentration of SDS, 
but not significantly. Less SDS leads to a slightly wider size distribution and larger particles. However, NP diameter distributions are all centered around $20 \mathrm{~nm}$. More SEM images can be found in Figure S1-S4.
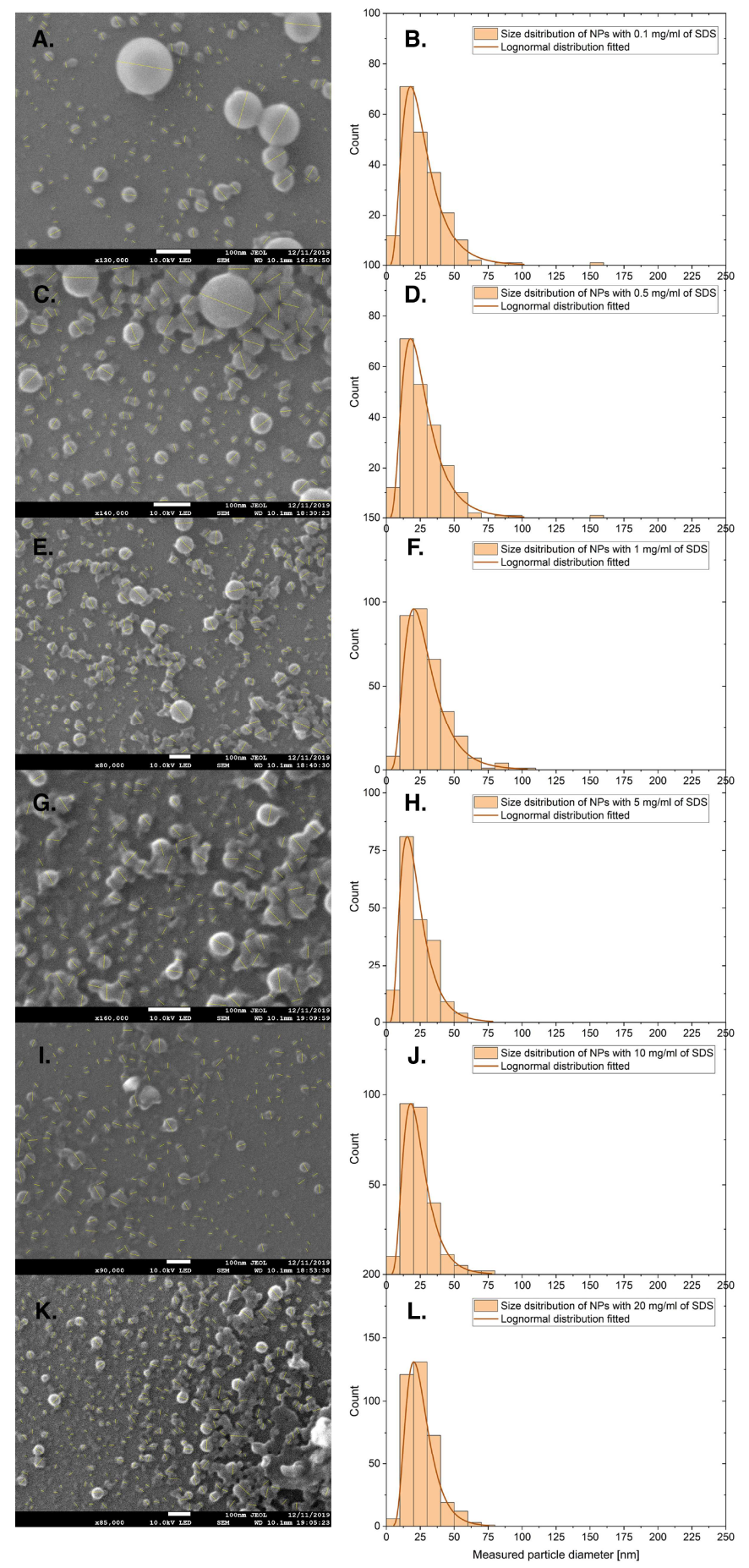

Figure 1: SEM images and corresponding size distributions for PS NPS with PDI at various SDS concentrations $\mathrm{AB}$ ) $0.1, \mathrm{CD})$ 0.5, EF) $1.0 \mathrm{GH}) 5.0, \mathrm{IJ}) 10$ and $\mathrm{KL}$ ) $20 \mathrm{mg} / \mathrm{ml}$. PS NPs were prepared according to the standard protocol with PS (Mw, 35,000) $1 \% \mathrm{w} / \mathrm{w} \%$ in $2 \mathrm{~mL}$ toluene and corresponding amount of SDS in $14 \mathrm{~mL}$ water. Scale bars in the images are $100 \mathrm{~nm}$. 


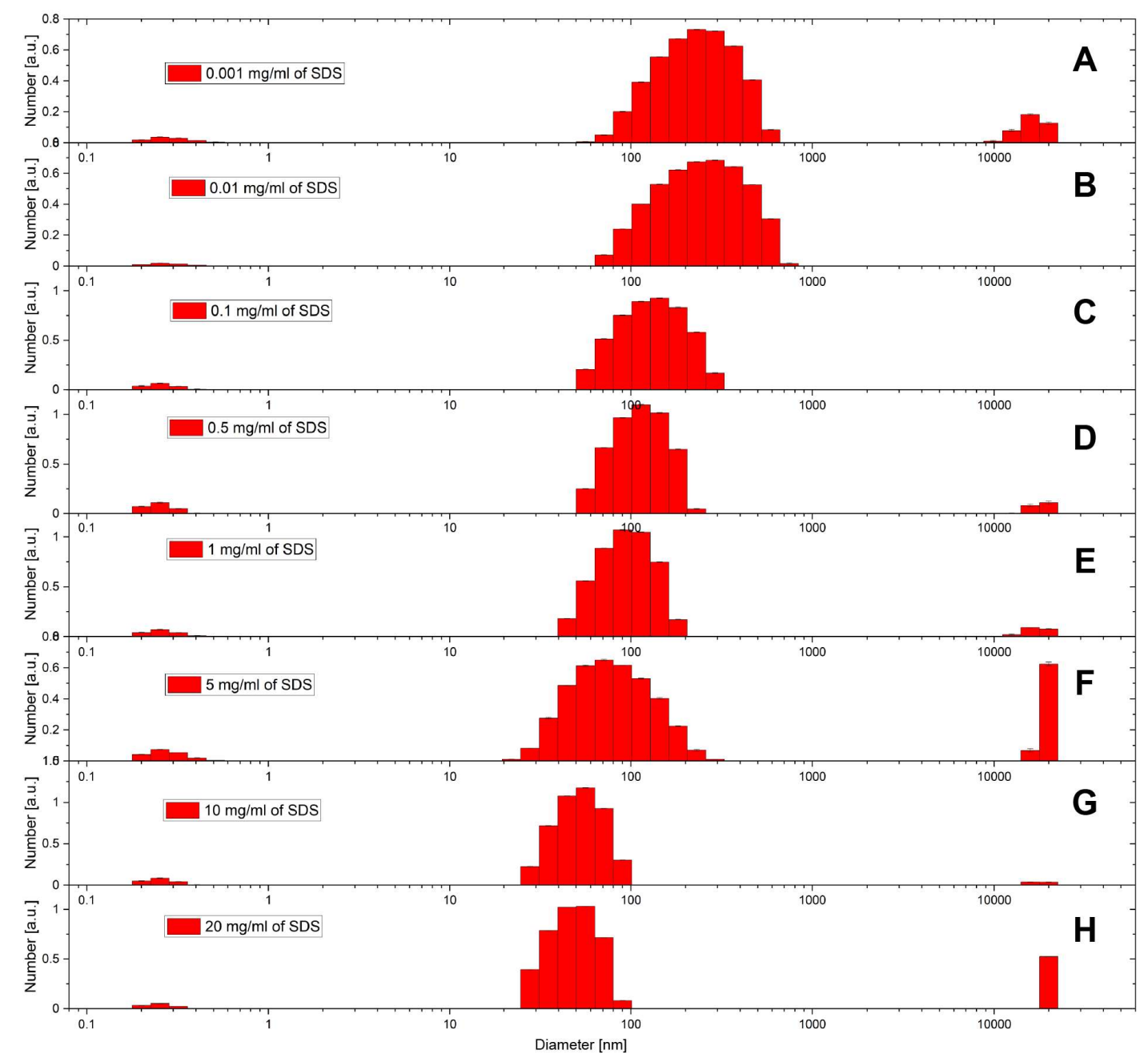

Figure 2. DLS results of size distribution for PS NPs with PDI at various SDS concentrations: A) 0.001 , B) 0.01 , C) 0.1 , D) 0.5, E) 1.0, F) 5.0, G) 10.0 and H) $20.0 \mathrm{mg} / \mathrm{ml}$. PS NPs were prepared according to the standard protocol with PS ( $\mathrm{Mw}, 35,000) 1 \% \mathrm{w} / \mathrm{w} \%$ in $2 \mathrm{~mL}$ toluene and corresponding amount of SDS in $14 \mathrm{~mL}$ water.

DLS was also used to evaluate the size distribution of the particles. Figure 2 shows the DLS results for the size distribution for the same batches of PS NPs with SDS concentrations varying from $1 \mu \mathrm{g} / \mathrm{ml}$ to $20 \mathrm{mg} / \mathrm{ml}$. Compared with SEM results, the DLS analysis results in larger diameters for PS NPs and also show palpable variations from $50 \mathrm{~nm}$ to $250 \mathrm{~nm}$. SEM works by measuring the conductivity of the dried sample surface and converts it to the geometry signal and therefore provides the physical size of the solid particles. DLS measures the scattering light changing with the movement of the particles, which are covered stationary double-layers. Thus, the observed differences between DLS and SEM can have two origins.

The first explanation is related to surface charge. Figure $\mathbf{2}$ includes the variations in the DLS results as the SDS concentration is decreased. This change can be caused by the changing of surface charge. As SDS is an ionic compound, a higher SDS level produces particles with more charge without considering the solid core size difference. Particles with higher zeta-potential tend to stay separately with high electrostatic repulsion among each other, while low charged particles have more risk to precipitate, 
which benefits the larger diameter result from DLS. This would lead to the observed trend in size distribution.

The second, trivial, explanation is that the larger NPs are underrepresented in the SEM images used to generate the size distribution plots. As multiple syntheses, SEM sample preparations, and several images gave rise to the same size distributions, we consider this origin of the difference between SEM and DLS results to be less likely, and assume that the differences between SEM and DLS are due to aggregation.

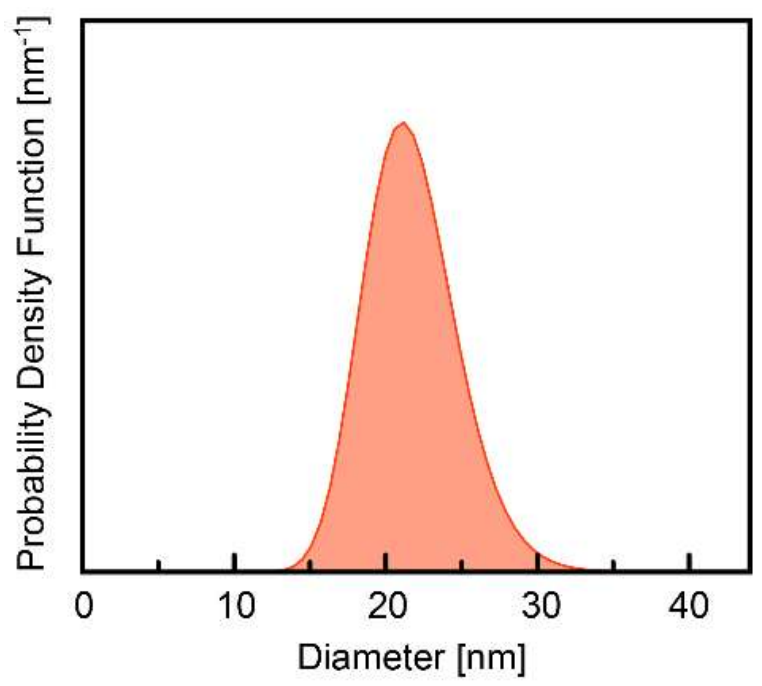

Figure 3. SAXS result of size distribution for standard PS NPs with DMQA. PS NPs were prepared according to the standard protocol with PS (Mw, 35,000) $1 \% \mathrm{w} / \mathrm{w} \%$ in $2 \mathrm{~mL}$ toluene and $1 \mathrm{mg} / \mathrm{ml}$ of SDS in $14 \mathrm{~mL}$ water.

To verify the obtained size distribution, a third method that probes the ensemble was used. Small angle X-ray scattering (SAXS) data were measured for the batch of standard PS NPs made with SDS, 1 $\mathrm{mg} / \mathrm{ml}$. The data was recorded on NPs isolated as a powder. Figure 3 shows the resulting size distribution that shows a mean diameter of $d=21 \mathrm{~nm}$. The size distribution obtained from SAXS analysis is similar to the one obtained from SEM. The SAXS ensemble size distribution is narrower than what is indicated by the size statistics from the SEM images (Figure 1F), where outliers are readily identified. All SAXS modeling details are included below and in Figure $\mathbf{S 5}$.

The confirmation of the size distribution by the SAXS experiment, allow us to conclude that SEM can be used as a representative tool to determine size distributions. Further we can conclude that the overall effect of the SDS concentration on the size distributions is small.

To probe the effect of surfactant concentration in detail and to facilitate screening, we adopted an easy and direct way that manually measure the largest and smallest NP observed in the SEM images with ImageJ. Figure 4 shows the largest and smallest particle size for the particle, with different amounts of SDS used in the synthesis. This analysis is more sensitive to changes than the overall size distribution. The data in Figure 4 shows that the smallest size does not change, it is limited by the resolution of SEM. The largest recorded NP size decreases with the concentration of SDS, but stops changing when the surfactant concentration reached the critical micelle concentration (CMC) of SDS $\left(8.08 \mathrm{mM}\right.$ or $2.33 \mathrm{mg} / \mathrm{mL}$ in water, $\left.25^{\circ} \mathrm{C}\right)$.[59] The inserted photo in Figure 4 shows that the NP synthesis solution is more transparent and creates more foam when stirred for solution with higher SDS concentration. 


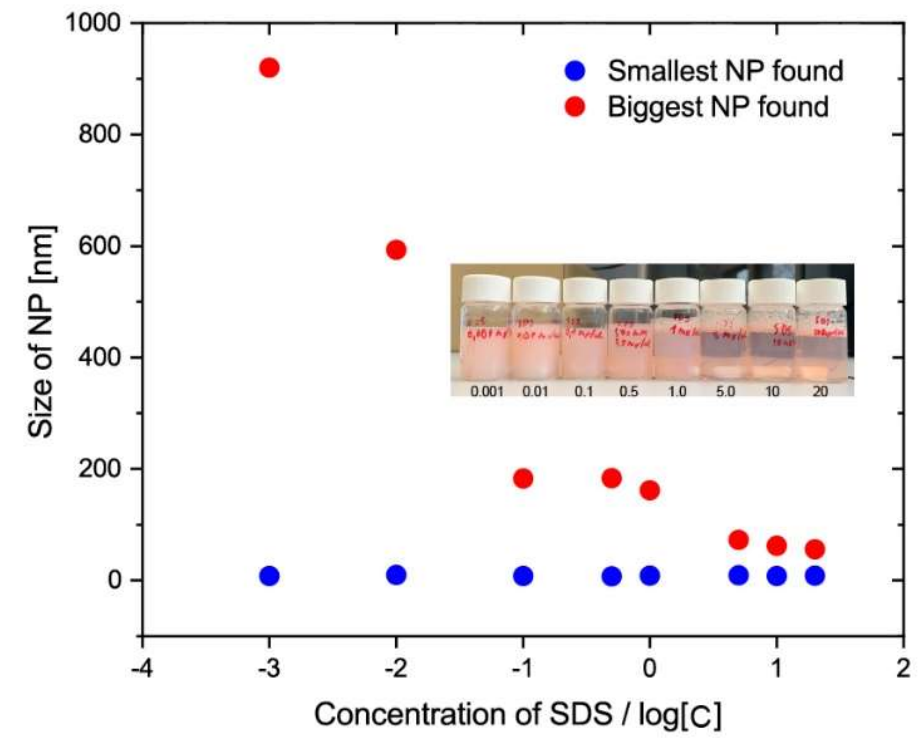

Figure 4: Size of largest and smallest observed nanoparticles in SEM images as a function of SDS concentration in $\mathrm{mg} / \mathrm{mL}$ plotted on semi-log plot. Samples were prepared according to the standard protocol with $2 \mathrm{~mL}$ of the organic phase (PS, Mw, 35,000 1\% w/w\% in toluene) and $14 \mathrm{~mL}$ water phase containing corresponding SDS concentration. Inserted photo is for the PS NPs with PDI at various SDS concentrations in $\mathrm{mg} / \mathrm{mL}$. The picture was taken before purification.

\section{Effect of the relative volume of toluene and concentration of polystyrene}

In addition to the surfactant concentration, the amount of the other ingredients should also influence the size distribution. Thus, we prepared the PS NPs by varying the volume of toluene from $1 \mathrm{~mL}$ to 4 $\mathrm{mL}$ to $14 \mathrm{~mL}$ of water, and the concentration of polystyrene in the toluene phase. The SDS concentration was kept constant at $0.01 \mathrm{mg} / \mathrm{mL}$ in each sample. We evaluated the effect using the largest and the smallest nanoparticles, see above. The data in Figure $\mathbf{5}$ shows that larger NPs can be found when the volume of the organic phase is increased, while a higher polystyrene concentration also gives a moderate increase in size. To ensure that it was not an effect related to a system where SDS was used as a surfactant, we also used Triton X-100, where the effects are the same (Figure S6). 

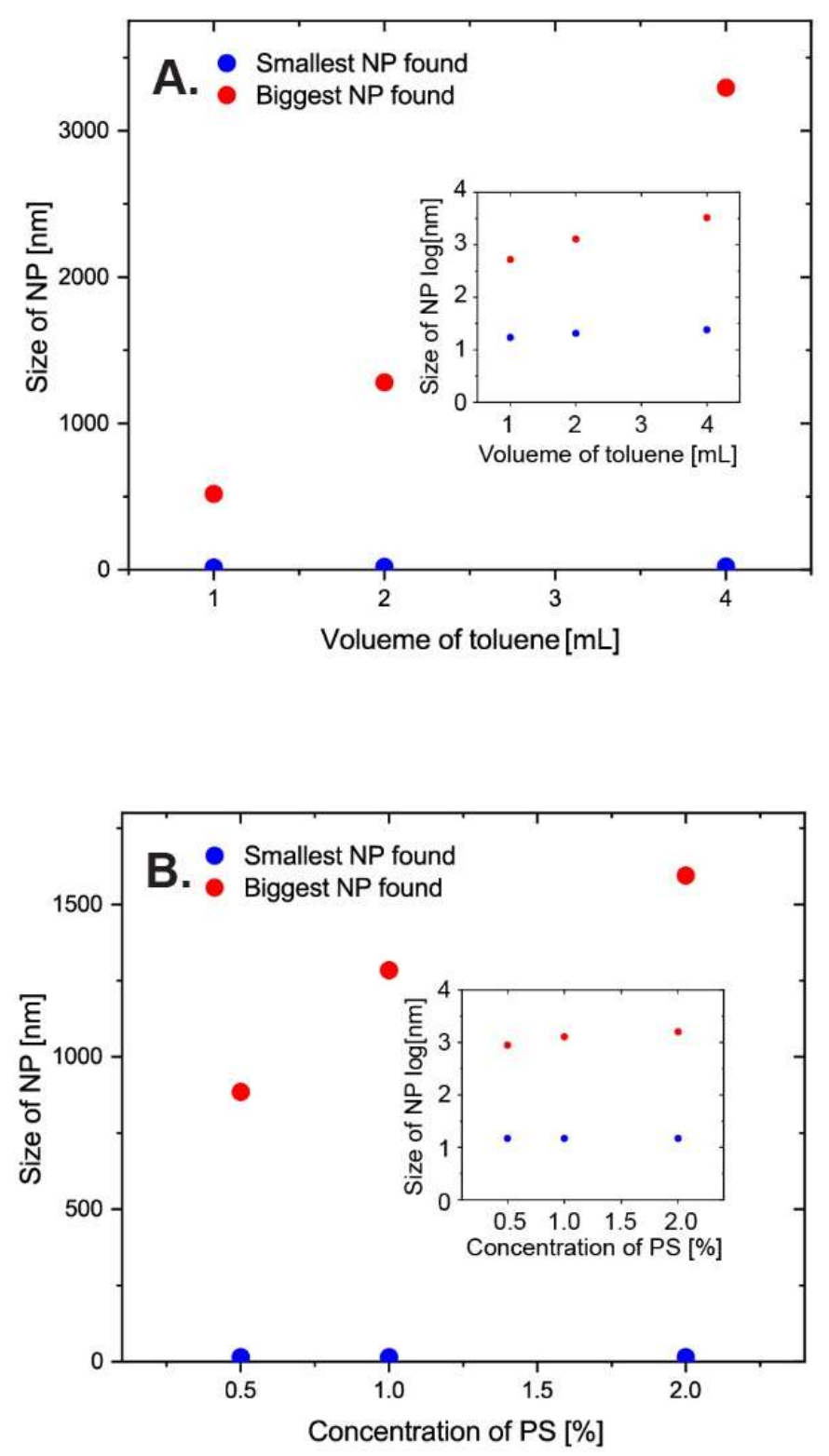

Figure 5. Size of largest and smallest observed nanoparticles in SEM images as a function of various amounts of A) toluene (PS NPs were prepared according to the standard protocol with $17.3 \mathrm{mg}$ of PS ( $\mathrm{Mw}, 35,000$ ) in $1 \mathrm{~mL}, 2 \mathrm{~mL}$ or $4 \mathrm{~mL}$ toluene; SDS $0.01 \mathrm{mg} / \mathrm{mL}$ in $14 \mathrm{~mL}$ of water) and B) polystyrene (PS NPs were prepared with respectively $0.5 \%, 1 \%$ or $2 \%$ of PS (Mw, 35,000) in $2 \mathrm{~mL}$ of toluene; SDS, $0.01 \mathrm{mg} / \mathrm{ml}$ in $14 \mathrm{~mL}$ of water). Respective data was plotted on semi-logarithmic axes in smaller graphs inserted.

\section{Effects of surfactant type and polystyrene chain length.}

Both foaming capacity and CMC value vary between different types of surfactants.[59-62] Surfactants will affect many characteristics of an emulsion including charge of NPs, the interfacial layer thickness, and the permeability.[63-65] In this work, two ionic (SDS and CTAB) and two non-ionic (Triton X-100 and Synperonic F108) surfactants (see the structures in Scheme S1) were used in the same molar 
concentration to fabricate four batches of PS NPs following the standard synthesis procedure, see below. The size of the largest and the smallest nanoparticles found with SEM are plotted in Figure 6A. SEM images are shown in Figure S7. NPs with SDS and CTAB show narrower size distribution than the NPs with Triton X-100 and Synperonic F108, and we must conclude that the type of surfactant is the one parameter probes so far that has a substantial effect on the resulting particle size, as well as the particle properties.
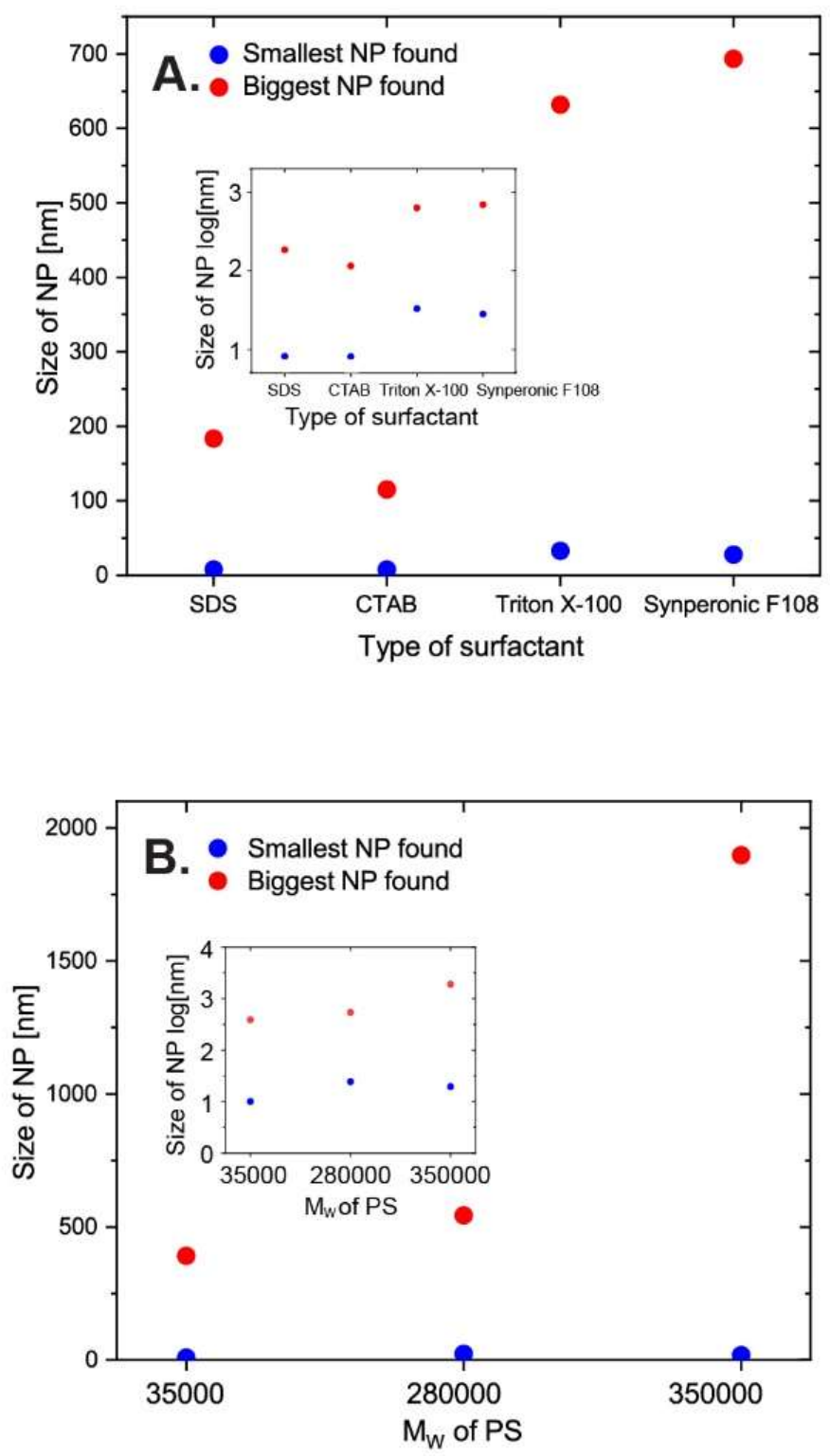

Figure 6. Size of largest and smallest observed nanoparticles in SEM images for samples prepared with A) $1.73 \mathrm{mM}$ of different surfactants (correspondingly SDS $0.5 \mathrm{mg} / \mathrm{mL}$, CTAB $0.6 \mathrm{mg} / \mathrm{mL}$, Triton X-100 $1.1 \mathrm{mg} / \mathrm{mL}$, Synpernoic $25 \mathrm{mg} / \mathrm{mL}$ in $14 \mathrm{~mL}$ of water; PS (Mw, 35,000) 1\%, w/w\% in $2 \mathrm{~mL}$ of toluene; with the standard protocol) or B) polystyrenes with Mw respectively 35,000, 280,000, and 350,000 (1\%, w/w\% PS in $2 \mathrm{~mL}$ of toluene; SDS $1.0 \mathrm{mg} / \mathrm{mL}$ in $14 \mathrm{~mL}$ of water; with the standard protocol). Respective data was plotted on semi-logarithmic axes in smaller graphs inserted. 
The molecular weight of the polystyrene can vary in several orders of magnitude, so we also considered this as a factor that influences the size distribution. We prepared the PS NPs with the standard method but with different kinds of PS (Mw, 35,000, 280,000, and 350,000) under the same weight percentage (1\%). Figure 6 B shows the size of the biggest and the smallest nanoparticles found with SEM (Figure S8ABC ). We could find bigger NP for the sample prepared with a bigger molecular weight. DLS results are coincident with the SEM results showing mean diameters of the three batches of $48 \mathrm{~nm}, 68 \mathrm{~nm}$, and $74 \mathrm{~nm}$ (Figure S10A), and they are not significantly different. Additionally, SAXS data were collected for these 3 batches. Unfortunately, the SAXS data could not be well modeled because the particles agglomerated into an ordered structure, with repeating distances of $18 \AA$ and $36 \AA$ (Figure S11). However, the scattering patterns are similar to those obtained from the less ordered particles (Figure S11, 35,000_DMQA and Figure S5B), which means all the type of this PS-vary samples have size distributions that are close to the previous batch (SDS, $1 \mathrm{mg} / \mathrm{ml}$; dye, DMQA). The molecular weight of PS does influence the size distribution, and larger Mw leads to larger particles.

\section{Final procedure optimization and scale-up}

We also tried to optimize the standard synthesis procedure by adjusting the number of refinements (sonication cycles)and duration between the refinements. A standard synthesis procedure was followed to prepare six samples where only the conditioning durations between the refinements were adjusted respectively to $15,30,45,60,75$, and $90 \mathrm{~min}$. The smallest and the largest nanoparticles of each sample were observed with SEM. Figure S12A shows no obvious difference when we tune the conditioning time between refinements. Different numbers (1 to 5 ) of refinement were also tested if it affected the properties of the NPs. Figure S12B shows the largest and the smallest nanoparticles observed for the samples prepared with the standard mixture of reagents $(2 \mathrm{~mL}$ organic phase / 14 $\mathrm{mL}$ water phase) with different times of refinements. No significant change was found. The photo in Figure S12C shows fewer times of sonicating leads to more PS toluene solution left as a separate layer. Although SEM results do not indicate a significant difference in micro scale, 5 times is considered to be preferable to make full use of the polystyrene solution by ensuring that all of it is in the suspension.

In order to check the repeatability of this standard synthesis method, we fabricated another three batches of PS NP with respectively three different PS (Mw, 35,000, 280,000,350,000) using $1 \mathrm{mg} / \mathrm{mL}$ SDS as the surfactant. SEM images in Figure S8DEF, DLS (Figure S10B), and SAXS (Figure S12) show how their size distributions are similar to the previous 3 batches (PS, Mw, 35,000, 280,000, 350,000) (Figure S9). Two batches of PS NP were prepared with PS (Mw, 35,000) and respectively CTAB and Triton X-100 as surfactants using the standard method. From SAXS (Figure S11), we observe how PS NP made from Triton X-100 shows a larger diameter while the one made from CTAB, which has similar size distribution as NPs prepared using the SDS. They are in agreement with the previous result, so our standard synthesis method is also repeatable for other surfactants.

A five-times larger preparation for the PS NP (PS, Mw, 35,000; SDS, $1 \mathrm{mg} / \mathrm{ml}$ ) was prepared with the standard synthesis method. DLS shows the hydrodynamic radius is $66 \mathrm{~nm}$ which is larger than the NPs prepared in the normal scale ( $24 \mathrm{~nm}$ from Figure S10). While SEM (Figure S13 \& S9) and SAXS (Figure S11) results proved the size distribution is similar to the batch with normal amount ingredients.

\section{Functionalization of PS NPs with dye loading}

Most of the lipophilic fluorescence dyes can be quenched by water. In PS NPs the majority of the population of the fluorophores will be contained inside of the nanoparticle rather than on the surface, giving rise to stronger fluorescent signal. We tested the encapsulation of four dyes (PDI, DMQA, thioxanthene-9-one, and celestine blue, Scheme S2) using the standard preparation protocol. Fluorescence spectra show these purified dye-doped PS NPs in water solution are highly fluorescent 
with spectra similar to the dyes dissolved in organic solvents. DMQA doped PS NPs have been characterized and used as a reference dye for the $\mathrm{pH}$ optical sensor in our lab (Figure S14).[70] Figure 7 shows the fluorescence emission and excitation spectra, size distribution, and some SEM images for the thioxanthene-9-one doped PS NPs. Average diameter is $25 \mathrm{~nm}$ for these dyes doped NPs and size distribution does not change when the particles are doped with dyes.
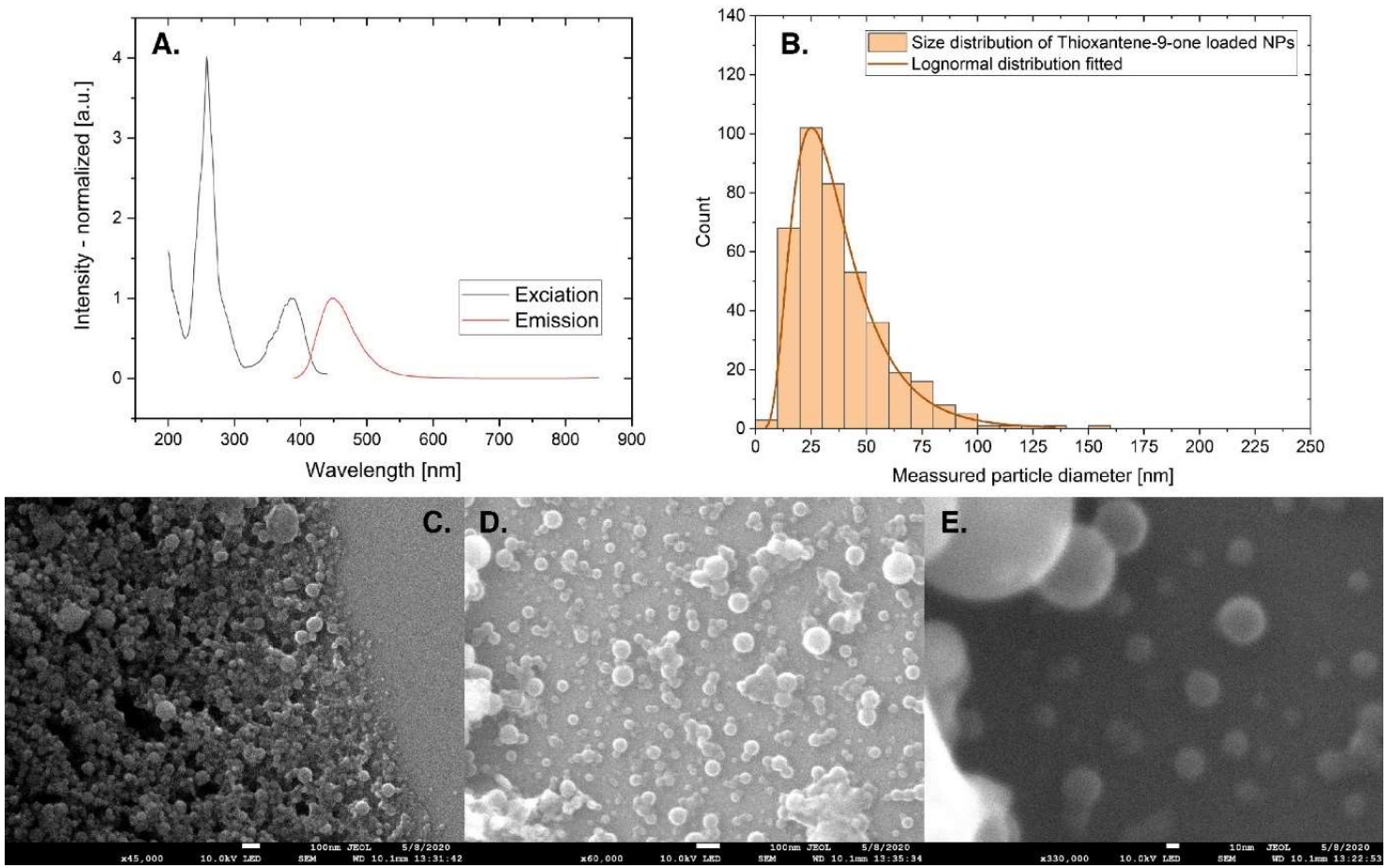

Figure 7. A) Excitation and emission spectra, B) size distribution counted from SEM image $D(n=397$, bin size $10 \mathrm{~nm}$ ) and CDE) SEM images for PS NPs loaded with Thioxantene-9-one.

\section{Discussion}

Table 1. The summary for the effects of all the variable factors

\begin{tabular}{|c|c|c|c|c|}
\hline \multirow[t]{2}{*}{ Name of a variable } & \multirow{2}{*}{$\begin{array}{l}\text { Magnitude of } \\
\text { the effect }\end{array}$} & \multicolumn{2}{|c|}{ Size of PS NPs } & \multirow[t]{2}{*}{ Notes } \\
\hline & & Increase & Decrease & \\
\hline Increasing surfactant concentration & medium & & $\mathrm{v}$ & - \\
\hline Increasing volume of toluene & strong & $\mathrm{v}$ & & - \\
\hline Increasing concentration of PS & medium & $\mathrm{v}$ & & - \\
\hline Type of surfactant & strong & - & - & Surfactant dependent \\
\hline Increasing of PS molecular weight & medium & $v$ & & \\
\hline Increasing number of refinements & low & - & - & No apparent effect observed \\
\hline Increasing time between refinements & low & - & - & No apparent effect observed \\
\hline Addition of dye & low & - & - & No apparent effect observed \\
\hline
\end{tabular}


We have prepared more than 20 batches of pure and dye doped polystyrene nanoparticles. In addition to these preparations, where parameters were varied, three individual researchers have independently prepared batches of dye doped PS NP using our standard procedure. All three obtained comparable results.

The knowledge gleaned from the collected experience is shown in Table 1. The ratio of the ingredients affects the size distribution, but the critical aspect is not the specific values used-volumes do not have to be measured to the third significant digit-but the nature of the chosen ingredients. The most prominent factors are the amount of polystyrene used, the nature of the surfactant, and the amount of toluene. Specifically, more PS, toluene or both lead to larger NPs and to wider size distribution.

The surfactant type could dramatically influence the size distribution, and higher concentrations of surfactant leads to smaller NPs and narrower size distributions to a certain point. Meanwhile the influence of the PS molecule weight can be ignored.

The procedure itself, such as the duration between refinements and times of sonication cycles, do not affect the largest or smallest size of NP. However, to make full use of the ingredients, 1-hour duration between refinements and 5 cycles of sonication are preferred.

Finally, we saw no effect of adding dyes to the PS NPs.

\section{Conclusions}

We proposed a simple fabrication method for making dye doped polystyrene nanoparticles with a physical diameter of 20-25 nm in a reproducible manner. Four different dyes were successfully doped into polystyrene nanoparticles, and three different scientists have repeatedly prepared nanoparticles with similar properties and size distributions. Thus, we conclude that we have developed and mapped a robust procedure for preparing dye doped polystyrene nanoparticles, which can be applied in many areas including optical sensors, cell tracking, and optical microscopy.

\section{Methods and Materials}

\section{Regents and materials}

Polystyrenes $(\mathrm{Mw}=35,000,280,000$ and 350,000), sodium dodecyl sulphate (SDS), cetyltrimetylammonium bromide (CTAB), Triton X-100, Synperonic F-108, toluene, thioxanthene-9one, celestine blue and the dialysis tube (cellulose membrane, avg. flat width, $10 \mathrm{~mm}$ ) were purchased from Sigma-Aldrich. N,N-bis(2,6-diisopropylphenyl)perylene-3,4:9:10-bis(dicarboximide) (PDI) was obtained from KU DYES, Denmark. N,N-(2-ethylhexyl)-1,13-dimethoxyquinacridinium hexafluorophosphate (DMQA) were synthesized in house.[66, 67] Milli-Q water was used for the preparation of the PS NPS.

\section{The standard PS NP synthesis procedure}

$14 \mathrm{mg} / 48.5 \mathrm{mmol}$ of SDS was dissolved in $14 \mathrm{ml}$ of water. $0.3 \mathrm{mg}$ of the desired dye was added to the PS toluene solution (17.34 $\mathrm{mg} 35.000 \mathrm{Mw}$ polystyrene in $2 \mathrm{~mL}$ toluene). The two solutions were mixed and the resulting two-phase system was sonicated (sonicator probe, Dia.: $7 \mathrm{~mm}$, sonicating power: 70 W) with pulsed sonication ( 1 second on and 1 second off) for a total duration of 60 seconds while the solution was stirred at $300 \mathrm{rpm}$. This sonication process was described as a refinement and was repeated 4 more times, 5 refinements in total. In between refinements, the vial was left stirring at 300 rpm with a closed cap for 60 minutes. After the final refinement, the sample was left stirring at 300 
rpm for 48 hours with an open cap, which allowed most of the toluene to evaporate. Extra surfactant was removed by dialysis. Dialyzed sample should not foam when shaken.

\section{Variations over the standard PS NP synthesis Procedure}

PS NPs were prepared with different surfactant concentrations. Different amount of SDS $(0.014,0.14$, $1.4,7,14,70,140$ or $280 \mathrm{mg}$ ) was dissolved in $14 \mathrm{ml}$ of water and $1 \%$ PS (Mw, 35,000) was prepared in $2 \mathrm{~mL}$ of toluene. For the rest of the synthesis procedure the standard process above was followed.

For the PS NPs prepared with different surfactants, $1.73 \mathrm{mM}$ of different surfactants (correspondingly SDS $0.5 \mathrm{mg} / \mathrm{mL}$, CTAB $0.6 \mathrm{mg} / \mathrm{mL}$, Triton X-100 $1.1 \mathrm{mg} / \mathrm{mL}$, Synpernoic $25 \mathrm{mg} / \mathrm{mL}$ ) was dissolved in $14 \mathrm{ml}$ of water and $1 \%$ PS (Mw, 35,000) was prepared in $2 \mathrm{~mL}$ of toluene. No dye was loaded. For the rest of the synthesis procedure the standard process above was followed.

For the PS NPs prepared with different PSs, the same weigh percentage $1 \%$ was used for each batch. SDS $(1 \mathrm{mg} / \mathrm{mL}$ in $14 \mathrm{~mL}$ water) was used for each sample. No dye was loaded. The rest of the process was identical to the standard procedure.

For the scaled-up PS NP batch, the organic phase was $10 \mathrm{~mL}$ of $1 \%$ PS (Mw, 35,000) toluene solution, while $70 \mathrm{~mL}$ of SDS $(1 \mathrm{mg} / \mathrm{mL})$ water solution was used as aqueous phase. No dye was loaded.The rest of the process was identical to the standard procedure.

\section{Instrumentation and measurements}

Scanning electron microscopic (SEM) images were obtained using a Jeol 7800F-prime scanning electron microscope $(10 \mathrm{kV})$ with a silicon wafer support. The samples were deposited on cleaned polished side of the silicon wafers and left them dry before the measurements.

For dynamic light scattering (DLS), an ALV/LSE-3 \& ALV-5000/60XO Correlator by ALV was used to measure the correlation function of the scattered laser intensity at $90^{\circ}$ from the beam path of a $630 \mathrm{~nm}$ Laser. The correlation function was then fitted using a regularized fit and then linear number weighted to yield the mean peak position. This was repeated for 5 runs per sample.

Small-angle X-ray scattering (SAXS) was measured on a SAXSLab instrument (JJ-X-ray, Denmark) at the Niels Bohr Institute, University of Copenhagen. PS NP solutions were dried by rotary evaporation at $36^{\circ} \mathrm{C}$ and the as-prepared powders were loaded in sandwich cells and measured at room temperature. The instrument is equipped with a 100XL + microfocus sealed X-ray tube from Rigaku that produces a photon beam with a wavelength of $\lambda=1.54 \AA$ and a 2D $300 \mathrm{~K}$ Pilatus detector from Dectris. The 2D scattering patterns were azimuthally averaged, normalised for sample transmission, primary beam intensity and exposure time and corrected for detector inhomogeneities using Saxsgui. The scattering angles, $\vartheta$, were converted to a $Q$-scale:

$$
Q=\frac{4 \pi}{\lambda} \cdot \sin (\theta)
$$

The data was fitted in Diffpy-CMI[68] and SASVIEW[69] with a power law to describe the long-range order of the particles and a spherical SAXS form factor, with a lognormal size-distribution, to describe the particle size and shape.

\section{Supporting information:}

Supporting Information File 1 
Schemes for chemical structures, SEM images and size analyzing from SEM, DLS, SAXS for PS NPS.

\section{Funding}

The authors thank Novo Nordisk Fonden (grant \#NNF190C0057136), Villum Fonden (grant \#14922 and grant \#15416), Carlsbergfondet and the University of Copenhagen for support. Mathilde Rove is thanked for her input to the final manuscript. A.S.A. acknowledge local support and continued access to the University of Copenhagen SAXSLab facility, in particular Erik Brok.

\section{References}

1. Frankær, C. G.; Hussain, K. J.; Rosenberg, M.; Jensen, A.; Laursen, B. W.; Sørensen, T. J. ACS Sensors 2018, 3 (3), 692-699.

2. $\quad$ Frankær, C. G.; Hussain, K. J.; Dorge, T. C.; Sørensen, T. J. ACS Sensors 2019, 4 (1), 26-31.

3. Carro-Temboury, M. R.; Arppe, R.; Vosch, T.; Sørensen, T. J. Science Advances 2018, 4 (1), e1701384.

4. Arppe-Tabbara, R.; Tabbara, M.; Sørensen, T. J. ACS Applied Materials \& Interfaces 2019, 11 (6), 6475-6482.

5. Sørensen, T. J.; Hildebrandt, C. B.; Elm, J.; Andreasen, J. W.; Madsen, A. O.; Westerlund, F.; Laursen, B. W. J. Mater. Chem. 2012, 22 (11), 4797-4805.

6. Feynman, R. P. Engineering and science 1959, 23.

7. $\quad$ Alivisatos, A. P. Science 1996, 271 (5251), 933-937.

8. Wang, X.; Zhuang, J.; Peng, Q.; Li, Y. D. Nature 2005, 437 (7055), 121-124.

9. Shen, J.; Sun, L. D.; Yan, C. H. Dalton Transactions 2008, (42), 5687-5697.

10. Guo, S. J.; Wang, E. K. Nano Today 2011, 6 (3), 240-264.

11. Biju, V. Chem. Soc. Rev. 2014, 43 (3), 744-764.

12. Peng, F.; Su, Y. Y.; Zhong, Y. L.; Fan, C. H.; Lee, S. T.; He, Y. Acc. Chem. Res. 2014, 47 (2), 612623.

13. Yao, J.; Yang, M.; Duan, Y. X. Chem. Rev. 2014, 114 (12), 6130-6178.

14. Lim, S. Y.; Shen, W.; Gao, Z. Q. Chem. Soc. Rev. 2015, 44 (1), 362-381.

15. Wolfbeis, O. S. Chem. Soc. Rev. 2015, 44 (14), 4743-4768.

16. Binderup, T.; Duivenvoorden, R.; Fay, F.; van Leent, M. M.; Malkus, J.; Baxter, S.; Ishino, S.; Zhao, Y.; Sanchez-Gaytan, B.; Teunissen, A. J. Science translational medicine 2019, 11 (506).

17. Peng, X. G.; Wickham, J.; Alivisatos, A. P. J. Am. Chem. Soc. 1998, 120 (21), 5343-5344.

18. Peng, X. G.; Manna, L.; Yang, W. D.; Wickham, J.; Scher, E.; Kadavanich, A.; Alivisatos, A. P. Nature 2000, 404 (6773), 59-61.

19. Bera, D.; Qian, L.; Tseng, T.-K.; Holloway, P. H. Materials 2010, 3 (4), 2260-2345.

20. Bourzac, K. Nature 2013, 493 (7432), 283.

21. Carey, G. H.; Abdelhady, A. L.; Ning, Z.; Thon, S. M.; Bakr, O. M.; Sargent, E. H. Chem Rev 2015, 115 (23), 12732-12763.

22. Reisch, A.; Didier, P.; Richert, L.; Oncul, S.; Arntz, Y.; Mély, Y.; Klymchenko, A. S. Nat. Commun. 2014, 5, 4089-4098.

23. Reisch, A.; Trofymchuk, K.; Runser, A.; Fleith, G.; Rawiso, M.; Klymchenko, A. S. ACS Applied Materials \& Interfaces 2017, 9 (49), 43030-43042.

24. Andreiuk, B.; Reisch, A.; Bernhardt, E.; Klymchenko, A. S. Chem. Asian J. 2019, 14 (6), 836-846.

25. Reisch, A.; Klymchenko, A. S. Small 2016, 12 (15), 1968-1992.

26. Chen, M. J.; Yin, M. Z. Prog Polym Sci 2014, 39 (2), 365-395.

27. Wu, C. F.; Schneider, T.; Zeigler, M.; Yu, J. B.; Schiro, P. G.; Burnham, D. R.; McNeill, J. D.; Chiu, D. T. J. Am. Chem. Soc. 2010, 132 (43), 15410-15417.

28. Grazon, C.; Rieger, J.; Meallet-Renault, R.; Charleux, B.; Clavier, G. Macromolecules 2013, 46 (13), 5167-5176. 
29. Tian, Z.; Shaller, A. D.; Li, A. D. Chem. Commun. 2009, 2 (2), 180-182.

30. Benson, C. R.; Kacenauskaite, L.; VanDenburgh, K. L.; Zhao, W.; Qiao, B.; Sadhukhan, T.; Pink, M.; Chen, J. S.; Borgi, S.; Chen, C. H.; Davis, B. J.; Simon, Y. C.; Raghavachari, K.; Laursen, B. W.; Flood, A. H. Chem 2020, 6 (8), 1978-1997.

31. Chen, J.; Fateminia, S. M. A.; Kacenauskaite, L.; Bærentsen, N.; Grønfeldt Stenspil, S.; Bredehoeft, J.; Martinez, K. L.; Flood, A. H.; Laursen, B. W. Angewandte Chemie International Edition 2021, 60 (17), 9450-9458.

32. Pelley, J. L.; Daar, A. S.; Saner, M. A. Toxicological Sciences 2009, 112 (2), 276-296.

33. Tsoi, K. M.; Dai, Q.; Alman, B. A.; Chan, W. C. Accounts Chem Res 2013, 46 (3), 662-671.

34. Valizadeh, A.; Mikaeili, H.; Samiei, M.; Farkhani, S. M.; Zarghami, N.; Akbarzadeh, A.; Davaran, S. Nanoscale research letters 2012, 7 (1), 480.

35. Wegner, K. D.; Hildebrandt, N. Chem Soc Rev 2015, 44 (14), 4792-4834.

36. Loos, C.; Syrovets, T.; Musyanovych, A.; Mailänder, V.; Landfester, K.; Nienhaus, G. U.; Simmet, T. Beilstein journal of nanotechnology 2014, 5 (1), 2403-2412.

37. Nasir, A.; Kausar, A. Polymer-Plastics Technology and Engineering 2015, 54 (17), 1819-1849.

38. Sapsford, K. E.; Algar, W. R.; Berti, L.; Gemmill, K. B.; Casey, B. J.; Oh, E.; Stewart, M. H.; Medintz, I. L. Chem Rev 2013, 113 (3), 1904-2074.

39. Ming, W.; Zhao, J.; Lu, X.; Wang, C.; Fu, S. Macromolecules 1996, 29 (24), 7678-7682.

40. Ming, W.; Jones, F. N.; Fu, S. Macromolecular Chemistry and Physics 1998, 199 (6), 1075-1079.

41. Zhang, C.; Wang, Q.; Xia, H.; Qiu, G. European polymer journal 2002, 38 (9), 1769-1776.

42. Xu, X.; Chew, C.; Siow, K.; Wong, M.; Gan, L. Langmuir 1999, 15 (23), 8067-8071.

43. He, G.; Pan, Q. Macromol Rapid Comm 2004, 25 (17), 1545-1548.

44. Fang, S.-J.; Fujimoto, K.; Kondo, S.; Shiraki, K.; Kawaguchi, H. Colloid and Polymer Science 2000, 278 (9), 864-871.

45. Ishii, H.; Ishii, M.; Nagao, D.; Konno, M. Polymer 2014, 55 (12), 2772-2779.

46. Alkadasi, N. A. N.; Al-Eyani, A. J. M. S.

47. Harkins, W. D. Journal of the American Chemical Society 1947, 69 (6), 1428-1444.

48. Nuruzatulifah, A.; Nizam, A.; Ain, N. N. Materials Today: Proceedings 2016, 3, S112-S119.

49. Landfester, K. Annu. Rev. Mater. Res. 2006, 36, 231-279.

50. Groeneveld, E.; de Mello Donegá, C. The Challenge of Colloidal Nanoparticle Synthesis. In Nanoparticles; Springer: 2014; pp 145-189.

51. Landfester, K. Angewandte Chemie International Edition 2009, 48 (25), 4488-4507.

52. Santra, S.; Tapec, R.; Theodoropoulou, N.; Dobson, J.; Hebard, A.; Tan, W. Langmuir 2001, 17 (10), 2900-2906.

53. Sanchez-Dominguez, M.; Boutonnet, M.; Solans, C. Journal of Nanoparticle Research 2009, 11 (7), 1823.

54. Destrée, C.; B.Nagy, J. Adv. Coll. Interface Sci. 2006, 123-126, 353-367.

55. Yabu, H.; Higuchi, T.; ljiro, K.; Shimomura, M. Chaos: An Interdisciplinary Journal of Nonlinear Science 2005, 15 (4), 047505.

56. Rao, J. P.; Geckeler, K. E. Progress in Polymer Science 2011, 36 (7), 887-913.

57. Walker, R. M.; Decker, E. A.; McClements, D. J. Journal of Food Engineering 2015, 164, $10-20$.

58. Zirak, M. B.; Pezeshki, A. Int J Curr Microbiol Appl Sci 2015, 4 (9), 924-32.

59. Fuguet, E.; Ràfols, C.; Rosés, M.; Bosch, E. Analytica Chimica Acta 2005, 548 (1-2), 95-100.

60. Bhattarai, A.; Shrivastav, G.; Adhikari, C. N. Bibechana 2014, 11, 123-127.

61. Tiller, G. E.; Mueller, T. J.; Dockter, M. E.; Struve, W. G. Anal Biochem 1984, 141 (1), $262-266$.

62. Gyulai, G.; Magyar, A.; Rohonczy, J.; Orosz, J.; Yamasaki, M.; Bősze, S.; Kiss, É. Express Polymer Letters 2016, 10 (3), 216.

63. Palanuwech, J.; Coupland, J. N. Colloids and Surfaces A: Physicochemical and Engineering Aspects 2003, 223 (1-3), 251-262.

64. Mcclements, D. J. Critical reviews in food science and nutrition 2007, 47 (7), 611-649.

65. Bouchemal, K.; Briançon, S.; Perrier, E.; Fessi, H. Int J Pharm 2004, 280 (1-2), 241-251. 
66. Hargenrader, G. N.; Weerasooriya, R. B.; Ilic, S.; Niklas, J.; Poluektov, O. G.; Glusac, K. D. ACS Applied Energy Materials 2018, 2 (1), 80-91.

67. Kacenauskaite, L.; Bisballe, N.; Mucci, R.; Santella, M.; Pullerits, T. n.; Chen, J.; Vosch, T.; Laursen, B. W. Journal of the American Chemical Society 2021, 143 (3), 1377-1385.

68. Juhas, P.; Farrow, C. L.; Yang, X.; Knox, K. R.; Billinge, S. J. L. Acta Cryst. 2015, 71 (Pt 6), 562568.

69. Proceedings of "software downloading for SASVIEW" http://www.sasview.org/ (accessed April 29, 2021).

70. Bartos D, Rewers M, Wang L, Sørensen TJ. Incorporating Fluorescent Nanomaterials in Organically Modified Sol-Gel Materials - Creating Single Composite Optical pH Sensors. ChemRxiv. Cambridge: Cambridge Open Engage; 2021; This content is a preprint and has not been peer-reviewed. 